2011 Shanghai Asia-Pacific School and Workshop on Gravitation International Journal of Modern Physics: Conference Series

Vol. 7 (2012) 148-157

(C) World Scientific Publishing Company

DOI: $10.1142 / \mathrm{S} 2010194512004217$

\title{
NUMERICAL INVESTIGATION OF FIVE-DIMENSIONAL GRAVITATIONAL COLLAPSES
}

\author{
HISA-AKI SHINKAI \\ Faculty of Information Science and Technology, Osaka Institute of Technology, \\ 1-79-1 Kitayama, Hirakata, Osaka 573-0196, Japan \\ and \\ Computational Astrophysics Laboratory, \\ Institute of Physical \&3 Chemical Research (RIKEN), \\ Hirosawa, Wako, Saitama 351-0198, Japan \\ shinkai@is.oit.ac.jp \\ YUTA YAMADA \\ Faculty of Information Science and Technology, Osaka Institute of Technology, \\ 1-79-1 Kitayama, Hirakata, Osaka 573-0196, Japan \\ yamada@is.oit.ac.jp
}

\begin{abstract}
We introduce our numerical studies of gravitational collapses in five-dimensional (5D) space-time, with a purpose of studying the cosmic censorship hypothesis and the hoop conjecture. The first model is the collapse of spindle matter which was performed by Shapiro and Teukolsky (1991) who announced an appearance of a naked singularity in 4D. Comparing with 4D cases, we found that 5D collapses proceed more rapidly, the final configurations tend to be spherical, and apparent horizon (AH) forms in wider parameter ranges. We also observed positive evidence for formation of a naked singularity in highly spindle cases as well. The second model is the formation of black-ring in 5D. Our code does not include angular momentum, but the model would be helpful for basic understandings. We constructed an initial data sequence with ring-shaped matter, and observed the topology of $\mathrm{AHs}$, if formed. We found a critical ring radius for ring-shaped $\mathrm{AH}$, and it suggests a dynamical transition of $\mathrm{AH}$ topology from ring-shaped to spherical. We demonstrate such an example in time evolution.
\end{abstract}

Keywords: General Relativity; Higher Dimensions; Black Holes; Numerical Relativity; Singularities

PACS numbers: 04.20.Dw, 04.20.Ex, 04.25.dc, 04.50.Gh

\section{Introduction}

Black-holes in higher dimensional space-time are extensively studied for a decade. One of the motivations is the so-called "large extra-dimensional models" as a consequence of brane-world pictures, which have changed our viewpoints for a way of understanding the fundamental forces. The scenarios of unifying the gravity at $\mathrm{TeV}$ scale open the possibility of verification of higher-dimensional space-time models at the CERN Large Hadron Collider (LHC). 
In $4 \mathrm{D}$ Einstein space-time, Kerr geometry is known to the unique stationary black-hole solution in the asymptotically flat boundary. If a gravitational collapse ends with a black-hole, then its horizon topology is $S^{3}$, and the only physical quantities which characterize the black-hole are mass and angular-momentum. On the other hand, in higher-dimensional space-time, this may not be realized. After the discovery of a torus black-hole ("black ring") solution" in 5D Einstein equation, quite rich structures are now available, including black Saturn and black di-rings (see a review ${ }^{2}$ for references). We are interested in their dynamical features, such as the formation processes, stabilities and late-time fate of higher-dimensional "black objects", and are developing numerical analyses.

We, in this article, report our numerical studies on gravitational collapses in five-dimensional axisymmetric space-time.

One of the topic is the cosmic censorship hypothesis $(\mathrm{CCH})^{3}$, which was originally proposed in $3+1$ dimensional GR. CCH states that collapse driven singularities will always be clothed by event horizon and hence can never be visible from the outside. Regarding to this issue, Shapiro and Teukolsky ${ }^{4}$ (ST91, hereafter) numerically demonstrated a counter example; a highly prolate matter collapse makes spacetime singular without forming an apparent horizon $(\mathrm{AH})$, that is, an appearance of a naked singularity. We compared the dynamics between 4D and 5D of this collapsing behavior ${ }^{5,6}$, which is the topic of $\S 3$ of this article. In $5 \mathrm{D}$, two axes can be settled as rotational symmetric axes, so that we also compare gravitational collapses in axisymmetry with those in "doubly"-axisymmetric space-time.

The second topic is Thorne's hoop conjecture ${ }^{7}$, which states that black hole horizons are formed when and only when a mass gets compacted into a small region. The result of ST91 is matched with this hoop conjecture. In D-dimensional cases, the measure 'hoop' is supposed to be replaced with a hyper-hoop $p^{8,9,10,11,12,13} ;(D-$ 3 ) dimensional area $V_{D-3}$ around a mass. We examine the validity of hyper-hoop conjecture in a 5D initial-data sequence, using minimum area around the matter. We found that the areal criteria matches with the appearance of AHs for spheroidal matter configurations, but not for ring configurations if we treat the conjecture for the necessary and sufficient criteria for the formation of $\mathrm{AH}$.

The third topic is the dynamics of black-ring. We found the critical ring radius for ring-shaped $\mathrm{AH}$ in the initial data sequence, and it suggests a dynamical transition of AH topology from ring-shaped to spherical. We demonstrate such an example in time evolution in non-rotating space-time. This will be described in $\S 4$.

\section{Numerical Methods}

We numerically constructed five-dimensional axisymmetric [symmetric on $z$-axis, $\mathrm{SO}(3)$ ] or doubly-axisymmetric [symmetric both on $x$ and $z$-axes, $\mathrm{U}(1) \times \mathrm{U}(1)$ ], asymptotically flat space-time (see Figure 1). For the comparison, we also performed four-dimensional axisymmetric space-time evolutions. 

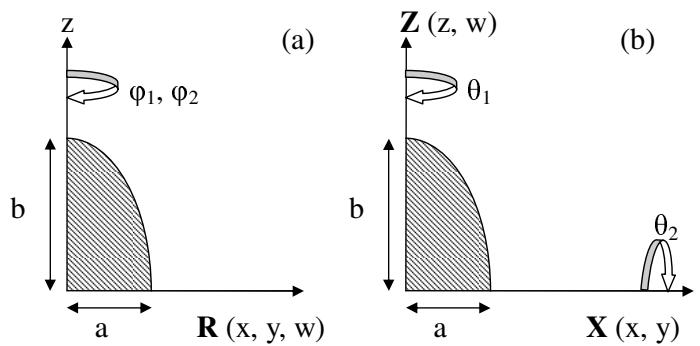

Fig. 1. We evolve five-dimensional (a) axisymmetric $[\mathrm{SO}(3)]$ or (b) double-axisymmetric $[\mathrm{U}(1) \times$ $\mathrm{U}(1)$, asymptotically flat space-time using the Cartesian grid. The initial matter configuration is expressed with parameters $a$ and $b$.

The asymptotical flatness is imposed throughout the evolution, which settles the fall-off condition to the metric as $\sim 1 / r$ for $4 \mathrm{D}$ cases and $\sim 1 / r^{2}$ for $5 \mathrm{D}$ cases.

We construct initial data sequences on a four-dimensional space-like hypersurface. Giving the matter distribution, the solution of the Einstein equations is obtained by solving the Hamiltonian constraint equation if we assume the moment of time symmetry. We apply the standard conformal approach ${ }^{14}$ to obtain the fourmetric $\gamma_{i j}$, with conformally flat trial metric. The details are described $\mathrm{in}^{5}$.

The space-time is evolved using the Arnowitt-Deser-Misner (ADM) evolution equations. It is known that the $\mathrm{ADM}$ evolution equations excite an unstable mode (constraint-violation mode) in long-term simulations ${ }^{15,16}$. However, we are free from this problem since gravitational collapse proceeds within quite short time. By monitoring the violation of constraint equations during evolutions, we confirm that our numerical code has second-order convergence, and also that the simulation continues in stable manner. The results shown in this report are obtained with numerical grids, $500 \times 500 \times 2 \times 2$.

The matter is described with 5000 collisionless particles, which move along the geodesic equations. We smooth out the matter by expressing each particle with Gaussian density distribution function with its typical width is twice as much as the numerical grid.

By imposing axisymmetry or double-axisymmetry, our model becomes practically a $(2+1)$-dimensional problem. We construct our numerical grids with the Cartesian coordinate $(x, z)$, and apply the so-called Cartoon method ${ }^{17,18}$ to recover the symmetry of space-time.

We use the maximal slicing condition for the lapse function $\alpha$, and the minimal strain condition for the shift vectors $\beta^{i}$ for spheroidal collapses, and zero-shift condition $\beta^{i}=0$ for ring collapses. Both conditions are proposed for avoiding singularities in numerical evolutions ${ }^{19}$, and the behavior of $\alpha$ and $\beta^{i}$ roughly indicates the strength of gravity, conversely. The iterative Crank-Nicolson method is used for integrating ADM evolution equations, and the Runge-Kutta method is used for matter evolution equations. 


\section{Spheroidal Collapse}

\subsection{Initial-data sequence}

We prepare several initial data fixing the total ADM mass and the eccentricity of distribution, $e=\sqrt{1-a^{2} / b^{2}}=0.9$ (see Figure 1 for $a$ and $b$ ). When the initial matter is highly prolate, $\mathrm{AH}$ is not observed. This is consistent with $4 \mathrm{D}$ cases ${ }^{20}$. $\mathrm{See}^{5}$ for details.

\subsection{Evolutions}

By changing the initial matter distribution sizes, we observe the different final structures. Figure 2 shows snapshots of $5 \mathrm{D}$ axisymmetric evolutions of model $b / M=4$ and 10 (model 5DS $\beta$ and $\operatorname{5DS} \delta$, respectively; see Table 1); the former collapses to a black hole while the latter collapses without forming an $\mathrm{AH}$.

All the models we tried result in forming a singularity (i.e., diverging $\mathcal{I}=$ $\left.R_{i j k l} R^{i j k l}\right)$. We stopped our numerical evolutions when the shift vector was not obtained with sufficient accuracy due to the large curvature. For model 5DS $\delta$, we integrated up to the coordinate time $t / M=15.4$ and the maximum of the Kretschmann invariant $\mathcal{I}_{\max }$ became $O(1000)$ on $z$-axis (see Figure $3($ Left)), but AH was not formed.

Table 1. Models and the results of their evolutions whether we observed $\mathrm{AH}$ or not. The eccentricity $e$ of the collapsed matter configurations is also shown; $e_{\mathrm{AH}}$ and $e_{\mathrm{f}}$ are at the time of $\mathrm{AH}$ formation (if formed), and on the numerically obtained final hypersurface, respectively.

\begin{tabular}{ccccc}
\hline$b / M(t=0)$ & 2.50 & 4.00 & 6.25 & 10.00 \\
\hline $4 \mathrm{D}$ axisym. & $4 \mathrm{D} \alpha$ & $4 \mathrm{D} \beta$ & $4 \mathrm{D} \gamma$ & $4 \mathrm{D} \delta$ \\
& AH-formed & no & no & no \\
& $e_{\mathrm{AH}}=0.90$ & & & \\
& $e_{\mathrm{f}}=0.92$ & $e_{\mathrm{f}}=0.89$ & $e_{\mathrm{f}}=0.92$ & $e_{\mathrm{f}}=0.96$ \\
\hline $5 \mathrm{D}$ axisym. & $5 \mathrm{DS} \alpha$ & $5 \mathrm{DS} \beta$ & $5 \mathrm{DS} \gamma$ & $5 \mathrm{DS} \delta$ \\
$\mathrm{SO}(3)$ & $\mathrm{AH}-$ formed & $\mathrm{AH}-$ formed & no & no \\
& $e_{\mathrm{AH}}=0.88$ & $e_{\mathrm{AH}}=0.88$ & & \\
& $e_{\mathrm{f}}=0.82$ & $e_{\mathrm{f}}=0.84$ & $e_{\mathrm{f}}=0.88$ & $e_{\mathrm{f}}=0.96$ \\
\hline $5 \mathrm{D}$ double & $5 \mathrm{DU} \alpha$ & $5 \mathrm{DU} \beta$ & $5 \mathrm{DU} \gamma$ & $5 \mathrm{DU} \delta$ \\
axisym. & $\mathrm{AH}-$ formed & $\mathrm{AH}-$ formed & $\mathrm{AH}-$ formed & no \\
$\mathrm{U}(1) \times \mathrm{U}(1)$ & $e_{\mathrm{AH}}=0.86$ & $e_{\mathrm{AH}}=0.87$ & $e_{\mathrm{AH}}=0.92$ & \\
& $e_{\mathrm{f}}=0.79$ & $e_{\mathrm{f}}=0.81$ & $e_{\mathrm{f}}=0.90$ & $e_{\mathrm{f}}=0.98$ \\
\hline
\end{tabular}

When the initial matter is highly prolate, $\mathrm{AH}$ is not observed. This is again consistent with 4D cases ${ }^{4}$, and agrees with the predictions from initial data analysis in $5 \mathrm{D} \operatorname{cases}^{5,9}$. The location of the 4 -dimensional Kretschmann invariant $\mathcal{I}_{\max }$ is on $z$-axis, and just outside of the matter. This is again the same with $4 \mathrm{D}$ cases $^{4}$. The absence of $\mathrm{AH}$ with diverging $\mathcal{I}$ suggests a formation of naked singularity in 5D. 
(a1)

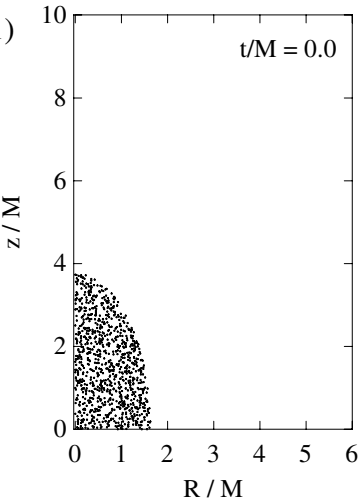

(a2)

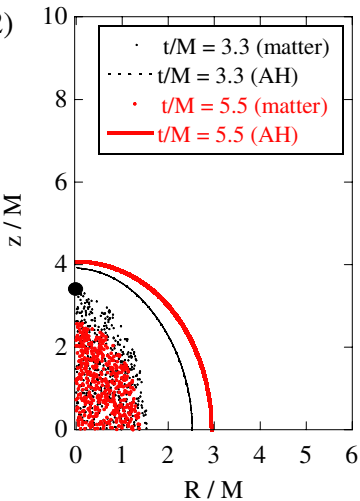

(b1)

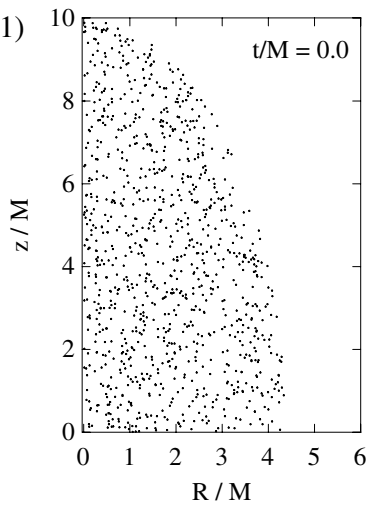

(b2)

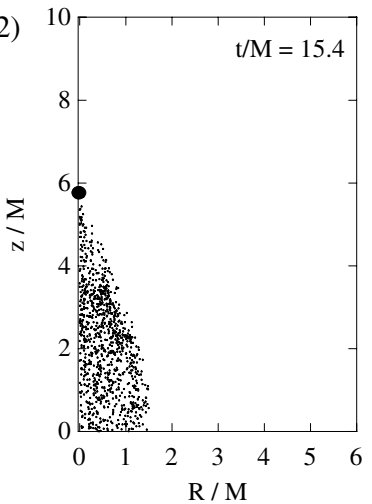

Fig. 2. Snapshots of 5D axisymmetric evolution with the initial matter distribution of $b / M=4$ [Fig.(a1) and (a2); model 5DS $\beta$ in Table 1] and 10 [Fig.(b1) and (b2); model 5DS $\delta$ ]. We see the apparent horizon $(\mathrm{AH})$ is formed at the coordinate time $t / M=3.3$ for the former model and the area of $\mathrm{AH}$ increases, while $\mathrm{AH}$ is not observed for the latter model up to the time $t / M=15.4$ when our code stops due to the large curvature. The big circle indicates the location of the maximum Kretschmann invariant $\mathcal{I}_{\max }$ at the final time at each evolution. Number of particles are reduced to $1 / 10$ for figures.

In order to compare the results with $4 \mathrm{D}$ and $5 \mathrm{D}$, we reproduced the results of ST91. We then find that the $e=0.9$ initial data with $b / M=10$ (model 4D $\delta$ ) collapses without forming $\mathrm{AH}$, and the code stops at the coordinate time $t=20.91$ with $\mathcal{I}_{\max }=84.3$ on the $z$-axis $(z / M=6.1)$; all the numbers match quite well with ST91. (Note that our slicing conditions and coordinate structure is not the same with ST91.)

We also performed 5D collapses with doubly-axisymmetric $[\mathrm{U}(1) \times \mathrm{U}(1)]$ spacetime. The matter and space-time evolve quite similar to 5D and 4D axisymmetric cases, but we find that the critical configurations for forming $\mathrm{AH}$ is different. Table 1 summarizes the main results of $4 \mathrm{D}$ and two 5D cases. We find that $\mathrm{AH}$ in $5 \mathrm{D}$ is formed in larger $b$ initial data than $4 \mathrm{D}$ cases. This result is consistent with our prediction from the sequence of initial data ${ }^{5}$. AH criteria with initial $b$ is loosened for 5D doubly-axisymmetric cases. 

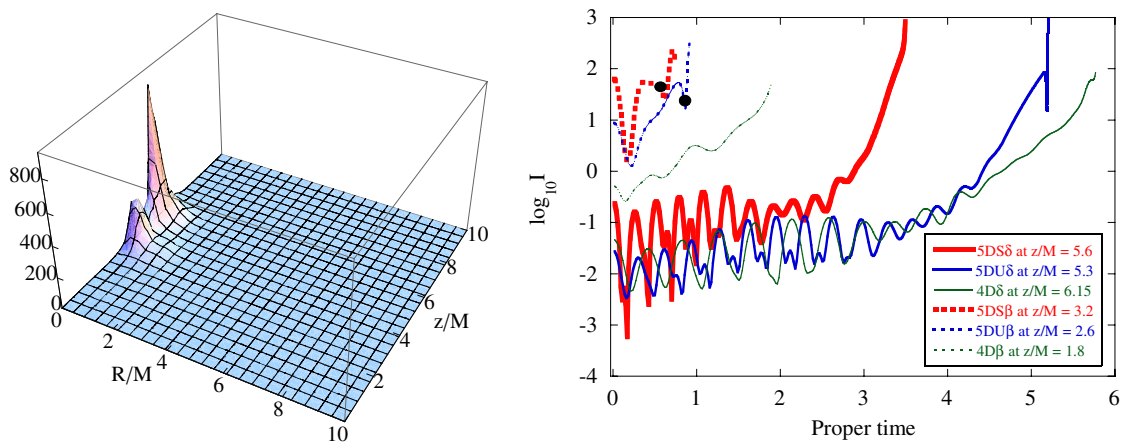

Fig. 3. (Left) Kretschmann invariant $\mathcal{I}$ for model $5 \mathrm{DS} \delta$ at $t / M=15.4$. The maximum is $O(1000)$, and its location is on $z$-axis, just outside of the matter. (Right) $\mathcal{I}$ at the location of $\mathcal{I}_{\max }$ on the final hypersurface is plotted as a function of proper time at its location. Labels indicate modelnames in Table 1. The time of $\mathrm{AH}$ formation ( $\mathrm{t}=0.6$ for model $5 \mathrm{DS} \beta, \mathrm{t}=0.9$ for $5 \mathrm{DU} \beta$ ) is shown by a dot.

We also show the eccentricity of matter, $e_{\mathrm{AH}}$ and $e_{\mathrm{f}}$, at the time of $\mathrm{AH}$ formation (if formed) and at the final time in the simulation, respectively. The numbers in Table 1 indicate that the eccentricity itself is not a guiding measure for $\mathrm{AH}$ formation, but they give us a hint for understanding the differences.

In $4 \mathrm{D}$, the eccentricity increases after $\mathrm{AH}$ is formed $(4 \mathrm{D} \alpha)$, while it decreases in $5 \mathrm{D}$ axisymmetric cases (5DS $\alpha$ and $5 \mathrm{DS} \beta$ ). That is, the $5 \mathrm{D}$ collapses proceed towards spherical configurations. This fact would be explained by the degree of freedom of the movements. In general, 5D space-time is expected to produce much gravitational radiation than $4 \mathrm{D}$ space-time $\mathrm{e}^{21,23}$, since there are more modes of oscillation exist. Gravitational radiation normally works to change shapes to spherical, because it is produced from the acceleration of space-time and carries the energy away. (It is known that compact binary system will evolve into a circular orbit due to the emission of gravitational radiation.) Therefore collapses in 5D space-time are likely to evolve towards more spherical. This interpretation together with the hoop conjecture will explain why AH-formation condition is loosened in $5 \mathrm{D}$ cases.

In the $5 \mathrm{D}$ doubly-axisymmetric cases, on the other hand, the magnitude of $e_{\mathrm{f}}$ is smaller than 5D axisymmetric cases for small $b / M$ cases (5DS $\alpha$ vs. 5DU $\alpha$, or 5DS $\beta$ vs. $5 \mathrm{DU} \beta$ ), while it is larger for large $b / M$ cases $(5 \mathrm{DS} \gamma$ vs. $5 \mathrm{DU} \gamma$, or $5 \mathrm{DS} \delta$ vs. $5 \mathrm{DU} \delta$ ). We think this is because that the doubly-axisymmetric collapses proceed in more symmetric manner than axisymmetric collapse near the origin, while they proceed in more 4D-like axisymmetric collapses near the axes far from the origin. The collapses of small $b / M$ initial data, therefore, will evolve into more spherical shape, while the large $b / M$ initial data will evolve increasing the eccentricity, where the latter is similar to $4 \mathrm{D}$ cases.

In Figure 3 (Right), we plot $\mathcal{I}$ at the point which gives $\mathcal{I}_{\max }$ on the final hypersurface as a function of proper time. The $\mathcal{I}$ diverges at the end of simulations in all the cases, but the diverging time becomes later for larger $b / M$ initial data. We see 
that 5D-collapses are generally proceeding more rapidly than $4 \mathrm{D}$ collapses. We also see that collapses in 5D doubly-axisymmetric space-time is proceeding more slowly than 5D single axisymmetric cases. If we observe further, the model 5DU $\beta$ evolves quite similar to $5 \mathrm{DS} \beta$, while $5 \mathrm{DU} \delta$ evolves quite similar to $4 \mathrm{D} \delta$. These behaviors support the previous understandings of the evolution of the eccentricity.

\subsection{Summary of spheroidal collapse}

The collapsing behaviors are generally quite similar to the cases in $4 \mathrm{D}$, but we also found that (a) 5D-collapses proceed rapidly than 4D-collapses, (b) AH appears in highly prolate matter configurations than $4 \mathrm{D}$ cases, (c) doubly-axisymmetric $[\mathrm{U}(1) \times \mathrm{U}(1)]$ assumption makes collapse proceed towards more spherical when it forms $\mathrm{AH}$, but presents quite similar behavior with 4D cases for large configurations, and (d) the positive evidence for appearance of a naked singularity in 5D.

\section{Ring Collapse}

\subsection{Initial-data sequence}

We prepare homogeneous toroidal matter configurations, described as

$$
\left(\sqrt{x^{2}+y^{2}}-R_{c}\right)^{2}+\left(\sqrt{w^{2}+z^{2}}\right)^{2} \leq R_{r}^{2}
$$

where $R_{c}$ is the circle radius of toroidal configuration, and $R_{r}$ is the ring radius. From obtained initial data, we also searched the location of AHs and the maximum value of the Kretchmann invariant $\mathcal{I}_{\max }$.

Fig. 4 shows two typical shapes of AHs. We set the ring radius as $R_{r} / r_{s}=0.1$ and search the sequence with changing the circle radius $R_{c}$, where $r_{s}$ is the Schwarzschild radius of the same mass. When $R_{c}$ is less than $0.78 r_{s}$, we find that only the $S^{3}-\mathrm{AH}$ ("common horizon" over the ring) exists. On the other hand, when $R_{c}$ is larger than $R_{c}=0.78 r_{s}$, only the $S^{1} \times S^{2}$-AH ("ring horizon", hereafter) is observed.

We find that $\mathcal{I}_{\max }$ appears at the outside of matter configuration. Interestingly, $\mathcal{I}_{\max }$ is not hidden by the horizon when $R_{c}$ is larger [see the case (c) of Fig.4].

We show the surface area of AHs $A_{3}$ in the left panel in Fig.5. We see typical two horizons monotonically decrease with $R_{c} / r_{s}$, the largest one is when the matter is spherical $\left(R_{c} / M=0\right)$. We also observe that the area of the common horizon is always larger than those of the ring horizon and both are smoothly connected in the plot. If we took account the analogy of the thermodynamics of black-holes, this suggests that the black-ring evolves to shrink its circle radius, and the ring horizon will switch to the common horizon at a certain radius in non-rotating space-time.

\subsection{Hyper-Hoop Conjecture}

We also calculate hyper-hoops which is defined by two-dimensional area. We propose to define the hyper-hoop $V_{2}$ as a surrounding two-dimensional area which satisfies 

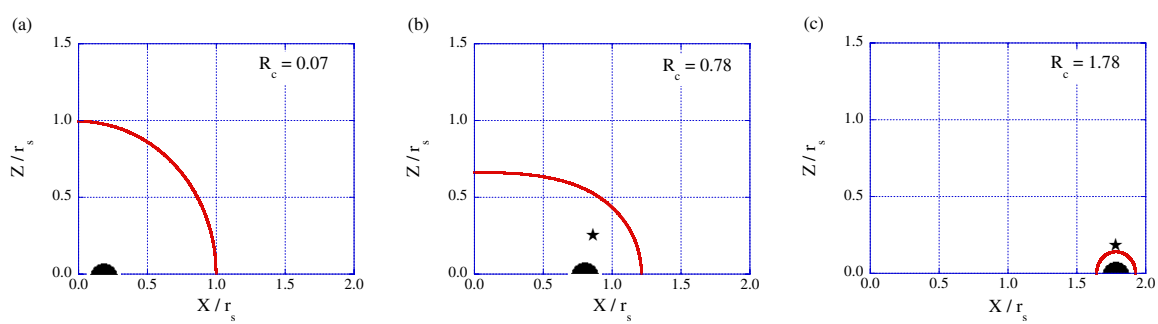

Fig. 4. Matter distributions (shaded) and the location of the apparent horizon (line) for toroidal matter configurations. The asterisk indicates the location of the maximum Kretchmann invariant, $\mathcal{I}_{\max }$.
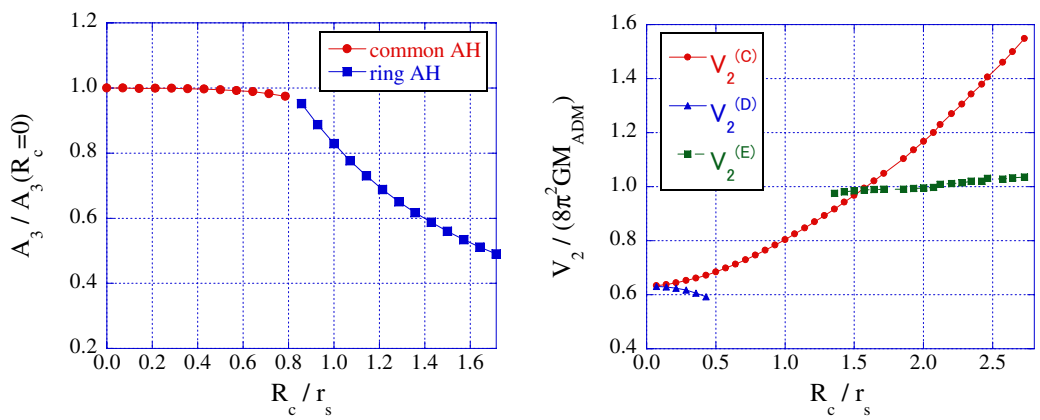

Fig. 5. (Left) The area of the apparent horizon $A_{3}$. Plots are normalized by the area of spherical case $\left(R_{c}=0\right)$. We see both horizons' area are smoothly connected at $R_{c} / r_{s}=0.78$, and both monotonically decrease with $R_{c} / r_{s}$. (Right) The ratio of the hyper-hoops $V_{2}$ to the mass $M_{A D M}$ are shown for the sequence of Fig.1. The ratio less than unity indicates that the validity of the hyper-hoop conjecture.

the local minimum area condition, $\delta V_{2}=0$. When the area of the space-time outside the matter is expressed with the coordinate $r$, then $\delta V_{2}=0$ leads to the EulerLagrange type equation for $V_{2}(r, \dot{r})$. We define the hoop $V_{2}^{(C)}$ and $V_{2}^{(D)}$;

Right panel in Fig.5 shows the hyper-hoops $V_{2}^{(C)}, V_{2}^{(D)}$, and $V_{2}^{(E)}$. We plot the points where we found hyper-hoops. We note that $R_{c} / r_{s}=0.78$ is the switching radius from the common horizon to the ring horizon, and that $V_{2}^{(C)}$ and $V_{2}^{(D)}$ are sufficiently smaller than unity if there is a common horizon. Therefore, hyper-hoop conjecture is satisfied for the formation of common horizon. On the other hand, for the ring horizon, we should consider the hoop $V_{2}^{(E)}$. In right panel of Fig.5, in the region $R_{c} / r_{s}>0.78, V_{2}^{(E)}$ exists only a part in this region and becomes larger than unity. Hence, for the ring horizon, the hyper-hoop conjecture is not a proper indicator as the necessary and sufficient conditions for its formation as far as our definition of the hyper-hoop is concerned. 


\subsection{Time Evolution}

Fig. 6 is a snapshot of evolutions for the ring matter of which initial radius are (a) $R_{c} / M=1.00$ and (b) 1.50 , respectively. Both have no AHs on the initial hypersurface, and we searched both spheroidal and toroidal horizons simultaneously at every time steps. We observe a formation of spheroidal AH (common horizon) in (a), while we see a formation of toroidal AH (ring horizon) then it switches to common horizon in (b).
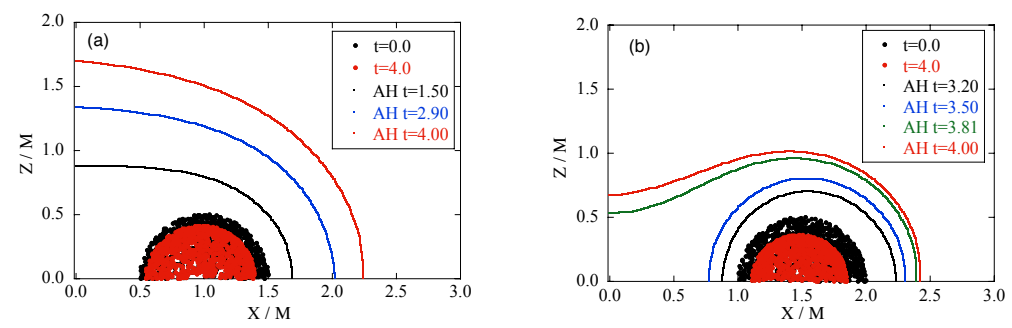

Fig. 6. Snapshots of evolutions of ring-shaped matter: for the initial data of (a) $R_{c} / M=1.00$ and (b) 1.50 , both with no AHs on the initial hypersurface. The matter distribution at $t=0.00$ and $t=4.00$, and the location of AHs are plotted on $X-Z$ coordinates. We see that AH topology switches from toroidal to spherical in (b).
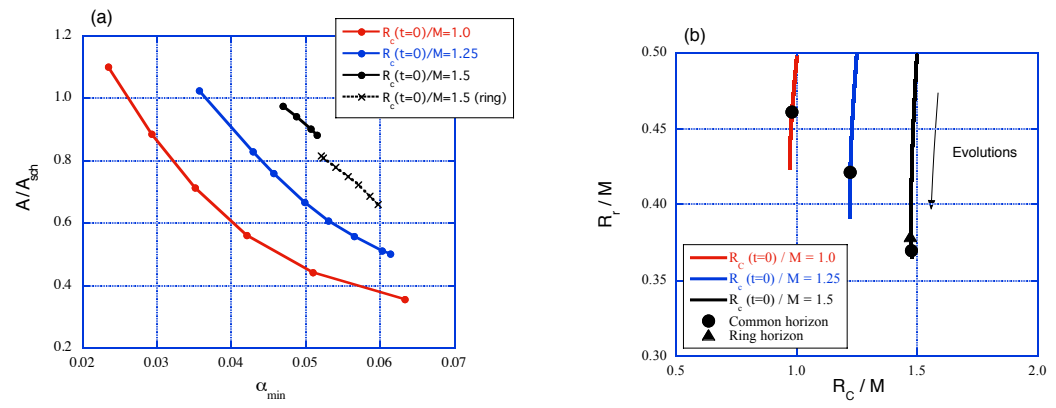

Fig. 7. (a) Area of $\mathrm{AH}$ is plotted as a function of minimum lapse function $\alpha_{\min }$ for three cases. We see AH increases monotonically, and jumps when the topology changes. (b) The ring matter configuration $\left(R_{c}, R_{r}\right)$ with marks when the horizon forms during its evolution.

In Fig. 7(a), we plot the $\mathrm{AH}$ area as a function of the minimum value of the lapse function, $\alpha$, which is located at the center of the matter and basically corresponds inversely with the maximum density of the matter. The area of $\mathrm{AH}$ is apparently increasing, and we see the area increases discontinuously when the topology of $\mathrm{AH}$ changes. We also plot the evolution of matter configuration $\left(R_{c}(t), R_{r}(t)\right)$ in 
Fig. 7(b) for the same 3 cases. The detail conditions for the formation of ring and common AHs will be reported elsewhere ${ }^{24}$.

\section{Future works}

In this article, we reported our numerical study of gravitational collapses in 5D space-time. We are now investigating the conditions for topology changes of AHs for ring matter evolutions, the relations to the hyper-hoop conjecture and/or Penrose inequality from dynamical viewpoints. Our code does not include any angular momentum, so that the actual studies of black-ring solution is not yet realized. We are implementing our code to cover these studies and hope to report them near future.

This work was supported partially by the Grant-in-Aid for Scientific Research Fund of Japan Society of the Promotion of Science, No. 22540293. Numerical computations were carried out on Altix3700 BX2 at YITP in Kyoto University, and on the RIKEN Integrated Cluster of Clusters (RICC).

\section{References}

1. R. Emparan and H. S. Reall, Phys. Rev. Lett. 88, 101101 (2002).

2. R. Emparan and H. S. Reall, Liv. Rev. Rel. 11, 6 (2008).

3. R. Penrose, Riv. Nuovo Cimento 1, 252 (1969).

4. S. L. Shapiro and S. A. Teukolsky, Phys. Rev. Lett. 66, 994 (1991).

5. Y. Yamada and H. Shinkai, Class. Quant. Grav. 27, 045012 (2010).

6. Y. Yamada and H. Shinkai, Phys. Rev. D. 83, 83, 064032 (2011).

7. K. S. Thorne, in Magic Without Magic, ed. by J. R. Klauder (Freeman, San Francisco), 231 (1972).

8. D. Ida and K. Nakao, Phys. Rev. D. 66, 064026 (2002).

9. C-M. Yoo, K. Nakao and D. Ida, Phys. Rev. D. 71, 104014 (2005).

10. C. Barrabés, V. P. Frolov, and E. Lesigne, Phys. Rev. D69, 101501 (2004).

11. J. M. M. Senovilla, Europhys. Lett. 81, 20004 (2008).

12. G. W. Gibbons, arXiv:0903.1580.

13. M. Cvetic, G.W. Gibbons, C.N. Pope, arXiv:1104.4504.

14. N. O Murchadha and J. W. York, Jr., Phys. Rev. D. 10, 428 (1974).

15. see e.g., H. Shinkai, J. Korean Phys. Soc. 542513 (2009).

16. H. Shinkai and G. Yoneda, Gen. Rel. Grav. 361931 (2004).

17. M. Alcubierre, S. Brandt, B. Bruegmann, D. Holz, E. Seidel, R. Takahashi, J. Thornburg, Int. J. Mod. Phys. D10, 273 (2001).

18. H. Yoshino and M. Shibata, Phys. Rev. D 80, 084025 (2009).

19. L. Smarr and J. W. York, Jr., Phys. Rev. D 17, 2529 (1978).

20. T. Nakamura, S. L. Shapiro and S. A. Teukolsky, Phys. Rev. D 38, 2972 (1988).

21. M. Shibata and H. Yoshino, Phys. Rev. D 81, 021501 (2010); Phys. Rev. D 81, 104035 (2010).

22. M. Zilhão, et al., Phys. Rev. D 81, 084052 (2010).

23. H. Witek, et al. Phys. Rev. D 82, 104014 (2010).

24. Y. Yamada and H. Shinkai, in preparation. 